NOTA CIENTÍFICA

\title{
Flora vascular y vegetación de los humedales de Puerto Viejo
}

\section{Vascular flora and vegetation from Puerto Viejo wetland}

\section{María I. La Torre y Héctor Aponte}

Museo de Historia Natural, Universidad Nacional Mayor de San Marcos, Avenida Arenales 1256, Jesús María, Lima. Apartado 14 Aponte: haponteu@yahoo.fr

\begin{abstract}
Resumen
El presente trabajo corresponde al primer reporte de flora vascular para el Humedal de Puerto Viejo, Cañete, Lima. Para esta localidad fueron registrados entre marzo del 2003 y julio del 2009, un total de 32 especies de plantas vasculares, agrupadas en 28 géneros y 15 familias. Las Magnoliópsida fueron el grupo dominante con 20 de los taxones. Del total de especies registradas el 34\% (11 taxones) corresponden a especies consideradas introducidas.
\end{abstract}

Palabras clave: Humedales, Cañete, costa peruana, flora vascular.

\section{Abstract}

Presentado: $\quad 04 / 11 / 2009$

Aceptado: $\quad 23 / 12 / 2009$

The present paper is the first list of vascular flora from the Puerto Viejo wetland, Cañete, Lima. A total of 32 species of vascular plants, grouped in 28 genera and 15 families were registered between March 2003 and July 2009. The Magnoliopsidae (Dicotyledoneae) was the dominant group with 20 taxa. The 34\% of the taxa (11 species) are considered as introduced species.

Keywords: wetland, Cañete, Peruvian coast, vascular flora.

\section{Introducción}

Los humedales son ecosistemas muy importantes no sólo por que albergan una gran diversidad de flora y fauna, sino también por los múltiples servicios ecosistémicos que brindan, como por ejemplo el control de los cursos de las corrientes de agua y la regulación del carbono global (Clarkson et al. 2004). Los humedales de la costa central del Perú, son parte de un corredor biológico dentro del desierto costero peruano-chileno. Gran parte de estos ecosistemas coexisten con poblaciones humanas, quienes hacen uso de los recursos que los humedales les proporcionan. Algunas actividades como la extracción del junco (Schoenoplectus americanus), la ganadería y usos inapropiados de los cuerpos de agua, han ocasionado que algunos de estos ambientes se vean deteriorados.

Al sur del departamento de Lima se encuentran el humedal de Puerto Viejo, un conjunto de cuerpos de agua de diferentes profundidades, en medio de un extenso gramadal y juncal, que recoge las afloraciones hídricas del acuífero del Río Mala. Este es uno de los ecosistemas menos protegidos de la costa y que ha recibido grandes impactos en los últimos diez años. A pesar de estar en proceso la categorización del área, hasta el momento no se cuenta con una lista oficial de plantas vasculares. El presente estudio aporta información actualizada de la riqueza florística de este ecosistema y sus formaciones vegetales.

\section{Área de estudio}

El Humedal de Puerto Viejo, se localizan al sur de Lima, en la provincia de Cañete en el distrito de San Antonio de Mala, a la altura del kilometro 71 de la panamericana Sur $\left(12^{\circ} 34^{\prime} \mathrm{S}\right.$ y $76^{\circ} 42^{\prime} \mathrm{W}$ ) a 22 metros sobre el nivel del mar (Fig. 1). Este humedal ha sido formado por las afloraciones hídricas del acuífero de Mala, que se distribuyen paralelamente al océano. Este humedal tiene aproximadamente 200 hectáreas y comprende varios cuerpos de agua zonas pantanosas y terrenos calcáreos. Los juncales y totorales que crecen en los bordes de estos cuerpos de agua son aprovechados para la extracción del junco y la totora (Typha dominguensis y Schoenoplectus californicus). Gran parte del humedal comprende un pastizal el cuál es utilizado para realizar actividades ganaderas (ovina y bovina).
El humedal se encuentra delimitado por el este con la carretera Panamericana Sur. Por el sur con una carretera afirmada que conduce a la zona de playa. Hacia el oeste se encuentra la zona Residencial Las Lagunas de Puerto Viejo. El Humedal es de propiedad privada. A pesar de ello cuenta con la categoría de Zona Reservada, sin que ningún plan de gestión por parte del Ministerio del Ambiente.
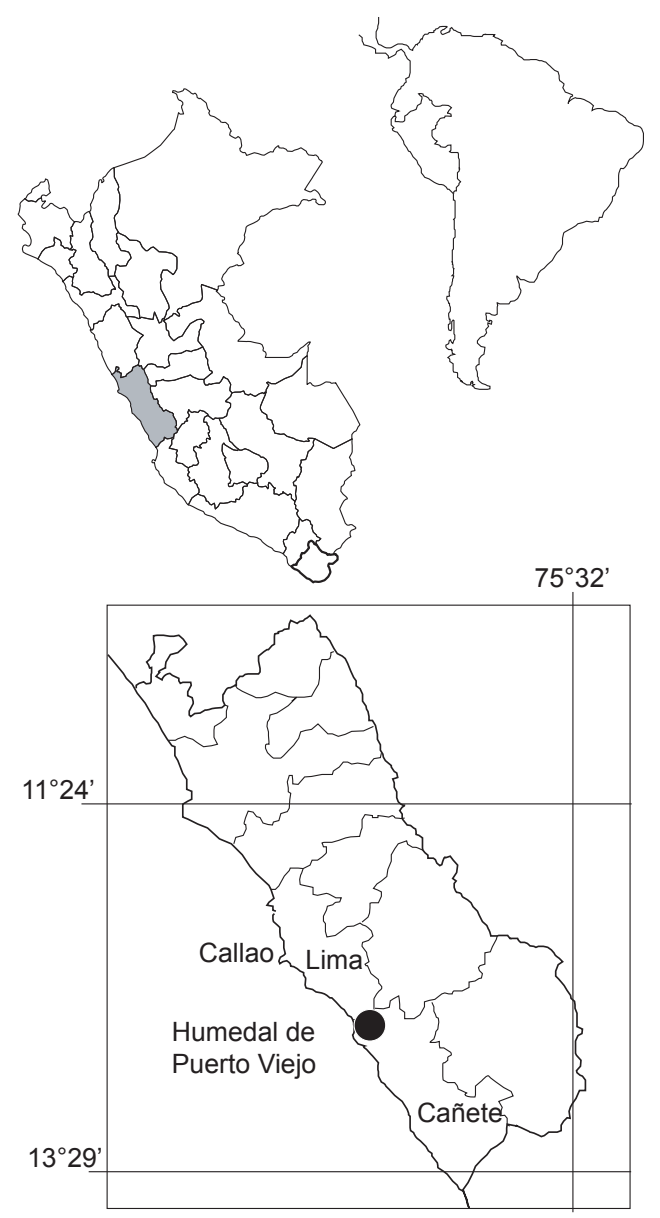

Figura 1. Mapa de Ubicación del Humedal de Puerto Viejo. En la esquina inferior izquierda se muestra un mapa del Perú donde se resalta el Departamento de Lima. 
Tabla 1. Lista de especies y Familias reportadas para el Humedal de Puerto Viejo. Se señala la $F C=$ forma de crecimiento $H=$ hierba; $A=$ arbusto, si la especie es considerada como introducida (IN) y si ha sido colectada en otros humedales $(\mathrm{OH})$

\begin{tabular}{|c|c|c|c|}
\hline Familias y especies & FC & IN & $\mathrm{OH}$ \\
\hline \multicolumn{4}{|l|}{ Aizoaceae } \\
\hline Sesuvium portulacastrum (L) L. & $\mathrm{H}$ & & $x$ \\
\hline \multicolumn{4}{|l|}{ Amaranthaceae } \\
\hline Alternanthera halimifolia. (Lamark) Standley ex Pittier. & $\mathrm{H}$ & $x$ & \\
\hline Alternanthera publifora (Bentham)Kuntze. & $\mathrm{H}$ & & \\
\hline \multicolumn{4}{|l|}{ Asteraceae } \\
\hline Encelia canescens. Lamark & $\mathrm{H}$ & & $x$ \\
\hline Pluchea chingoyo (H.BK)D.C. & A & & \\
\hline Acmella oleraceae (L.) R.K. Jansen & $\mathrm{H}$ & $x$ & \\
\hline Spilanthes leiocarpa DC. & $\mathrm{H}$ & $x$ & $x$ \\
\hline \multicolumn{4}{|l|}{ Bataceae } \\
\hline Batis maritima $\mathrm{L}$. & $\mathrm{H}$ & & \\
\hline \multicolumn{4}{|l|}{ Boraginaceae } \\
\hline Heliotropium curassavicum L. & $\mathrm{H}$ & & $x$ \\
\hline \multicolumn{4}{|l|}{ Chenopodiaceae } \\
\hline Chenopodium ambrosoides $\mathrm{L}$. & $\mathrm{H}$ & $x$ & \\
\hline Chenopodium murale $\mathrm{L}$. & $\mathrm{H}$ & & \\
\hline Sarcocornia neei (Lag.) M.A. Alonso \& M.B. Crespo. & $\mathrm{H}$ & & $x$ \\
\hline \multicolumn{4}{|l|}{ Cyperaceae } \\
\hline Bolboschoenus maritimus (L.)Palla & $\mathrm{H}$ & & \\
\hline Cyperus laevigatus L. & $\mathrm{H}$ & & $x$ \\
\hline Schoenoplectus americanus (Pers.) Volkart ex Schinz \& R. Keller & $\mathrm{H}$ & & $x$ \\
\hline Schoenoplectus californicus (C.A. Mey.) Soják & $\mathrm{H}$ & & $x$ \\
\hline \multicolumn{4}{|l|}{ Fabaceae } \\
\hline Melilotus indica (L) Allioni & $\mathrm{H}$ & $x$ & \\
\hline Prosopis pallida (Humbolt \& Bonpland ex & A & $x$ & \\
\hline \multicolumn{4}{|l|}{ Plantaginaceae } \\
\hline Plantago major $\mathrm{L}$ & $\mathrm{H}$ & $x$ & \\
\hline \multicolumn{4}{|l|}{ Poaceae } \\
\hline Arundo donax $\mathrm{L}$. & $\mathrm{H}$ & $\mathrm{X}$ & \\
\hline Cynodon dactylon (L.)Pers. & $\mathrm{H}$ & $\mathrm{X}$ & $\mathrm{X}$ \\
\hline Distichlis spicata (L)Greene & $\mathrm{H}$ & & $x$ \\
\hline Paspalidium geminatum (Forsskal)Stapf & $\mathrm{H}$ & & $x$ \\
\hline Polypogon viridis (Gouan) Breistr. & $\mathrm{H}$ & $x$ & $x$ \\
\hline Sporobolus virginicus (L) Kunt. & $\mathrm{H}$ & & $x$ \\
\hline \multicolumn{4}{|l|}{ Potamogetonaceae } \\
\hline Potamogeton striatus R\&P & $\mathrm{H}$ & & $x$ \\
\hline \multicolumn{4}{|l|}{ Scrophulariaceae } \\
\hline Baccopa monnieri (L) Pennell. & $\mathrm{H}$ & & $x$ \\
\hline \multicolumn{4}{|l|}{ Solanaceae } \\
\hline Lycium americanum Jacquin. & $\mathrm{H}$ & & \\
\hline Solanum americanum Miller & $\mathrm{H}$ & & $x$ \\
\hline Solanum pimpinellifolium L. & $\mathrm{H}$ & $x$ & \\
\hline \multicolumn{4}{|l|}{ Typhaceae } \\
\hline Typha dominguensis Persson & $\mathrm{H}$ & & $x$ \\
\hline \multicolumn{4}{|l|}{ Verbenaceae } \\
\hline Lippia nodiflora (L) Michaux & $\mathrm{H}$ & & $x$ \\
\hline
\end{tabular}

\section{Materiales y métodos}

Se realizaron colectas en los diferentes hábitats del humedal de acuerdo a técnicas estandarizadas (Bridson y Forman 1992), entre marzo del 2003 y julio del 2009. Se tomaron registro de las formas de crecimiento (Wittaker 1975). Asimismo, se registraron las coordenadas geográficas y altitudes mediante un receptor GPS. Los ejemplares colectados se encuentran depositados en el herbario San Marcos (USM). Las determinaciones taxonómicas fueron realizadas en el Laboratorio de Florística del
Museo de Historia Natural de la Universidad Nacional Mayor de San marcos, empleando claves y descripciones publicadas en literatura especializada (Sagastegui 1973; León 1993; Tovar 1993), además de consultas a especialistas. Las muestras fueron corroboradas con especímenes depositados en el herbario San Marcos (USM). Se consideraron como especies introducidas; a todas aquellas provenientes de otros habitas que no estén naturalizadas, a las especies exóticas, y aquellas consideradas como malezas (Sagástegui 1993). 


\section{Resultados}

La flora vascular del Humedal de Puerto Viejo está conformada por 32 especies de plantas vasculares, agrupadas en 28 géneros y 15 familias (Tabla 1). Las Magnoliópsida representan el $62 \%$ (20) de los taxones y las Liliópsida el 38\% (12 especies). Las familias más abundantes fueron las Poaceae (6), Cyperaceae(4), Asteraceae (4), Chenopodiaceae(3) y Solanaceae(3). La forma de crecimiento predominante son las hierbas, que constituyen el $94 \%$ de las especies totales (30 taxones). Del total de especies registradas el 34\% (11 taxones) corresponden a especies consideradas introducidas.

Fue posible identificar tres formaciones vegetales: Gramadal, Juncal y Totoral. El Gramadal está formado predominantemente por Distichlis spicata y Sarcocornia neei, que ocupa más de $60 \%$ del área total. El Juncal, compuesto por Schoenoplectus americanus, se encuentra en los bordes de los cuerpos de agua. Durante la época seca llegan a estas zonas plantas halófitas como diferentes especies de Chenopodium. El Totoral se encuentra igualmente en los bordes de los cuerpos de agua. Esta formación vegetal es de tamaño reducido, y está formado por las dos especie de totora reportadas para la zona: Schoenoplectus californicus y Typha dominguensis.

\section{Discusión}

La flora vascular que se reporta corresponde a múltiples años de trabajo, lo cuál nos ha permitido apreciar diversos cambios en la composición florística del área. Uno de los más resaltantes es probablemente la ausencia de especies acuáticas como Potamogeton striatus en las últimas colectas. Uno de los principales impactos en los cuerpos de agua de este humedal ocurrió en los años 90, cuando se inició la construcción de la zona residencial "Las Lagunas" y de forma paralela, la extracción de agua de las lagunas para el consumo en esta residencia. Este último hecho pudo haber afectado las poblaciones de plantas acuáticas en un inicio. Asimismo, en este humedal se han implementado zonas de extracción de totora balsa (Schoenoplectus californicus) por parte de los pobladores que viven en zonas aledañas, quienes dejan sus desechos en la zona de extracción, es decir, en la orilla de los lagos (Sanchez 2007). Este tipo de actividades puede modificar el nivel de nutrientes del agua, favoreciendo la aparición de algunas especies, pero también la desaparición de otras. Es así que en algunas zonas de extracción, se han encontrado especies oportunistas como Plantago major y Althernanthera halimifolia. Actividades como el monitoreo del estado de nutrientes de las lagunas serán necesarios para poder confirmar esta hipótesis. Esta actividad requiere igualmente de trabajo de capacitación de la población que extrae la totora.

En comparación con otros humedales de la costa central, Puerto Viejo presenta una mayor cantidad de especies que la laguna El Paraíso (25 especies) y el Área de Conservación Regional de Medio Mundo (16 especies), mientras que contiene una menor cantidad de especies que las reportadas para Pantanos de Villa (66 especies)(Cano et al. 1998). Cuando se comparan el número de especies compartidas por Puerto Viejo y las lo- calidades estudiadas anteriormente es posible percatarse que el $44 \%$ de las especies de Puerto Viejo (14 especies) no han sido reportadas anteriormente en listas de las otras localidades. La procedencia de estas especies particulares de Puerto Viejo es en su mayoría (8 especies) introducida. La actividad ganadera y los cambios en la calidad del agua pueden ser factores que podrían haber propiciado la aparición de estas especies. Poco se conoce acerca de la influencia de las vías de comunicaciones aledañas (carreteras) y de la ganadería en la composición florística del humedal. Un monitoreo constante y cuantitativo será necesario para lograr mayor resolución.

El presente trabajo corresponde al primer listado de plantas vasculares para esta área de estudio, que esperamos sea considerado para futuras actividades de gestión y conservación así como para la discusión científica de la composición florística de la costa del Perú.

\section{Agradecimientos}

Los autores agradecemos al Licenciado Robert Jiménez y su equipo por su apoyo y colaboración durante las colectas en la primera etapa del proyecto.

\section{Literatura citada}

Bridson, D. \& L. Forman. 1992 (eds.). The Herbarium Handbook. Royal Botanic Gardens. Kew.

Cano, A.; M.I. La Torre; B. León; K. Young; J. Roque \& M. Arakaki. 1998. Estudio comparativo de la Flora vascular de los Principales Humedales de las Zona Costera del Departamento de Lima, Perú. En: Los Pantanos de Villa: Biología y Conservación. A. Cano y K. Young (editores). Universidad Nacional Mayor de San Marcos (UNMSM). Serie de Divulgación $N^{\circ} 11$. pp. $181-190$.

Clarkson, B. R. ; B. Sorrell; P. Reeves; P. Champion; T. Patridge \& B. D. Clarkson. 2004. Handbook for Monitoring Wetland Conditions. Ministry for the Environment Sustainable Management Fund Project (5105).

León B. 1993. Catálogo anotado de las fanerógamas acuáticas del Perú. En: F. Kahn, B. León \& K.R. Young (eds.), Las Plantas Vasculares en las Aguas Continentales del Perú. Travaux de l'Institut Francais d'Etudes Andines Tomo 75. IFEA (Institut Francais d'Etudes Andines), Lima-Peru. $357 \mathrm{pp}$.

Sagástegui, A. 1973. Manual de las Malezas de la Costa Norperuana. Primera Edición 1973. Universidad Nacional de Trujillo. Trujillo, Peru. $480 \mathrm{pp}$

Sagástegui, A. \& Leiva S. 1993. Flora Invasora de los Cultivos del Perú. Consejo Nacional de Ciencia y Tecnología. Primera Edición. Trujillo, Perú. 539pp.

Sánchez R 2007. Informe técnico del aprovechamiento del junco en las Humedales de la Costa central del Perú. En: Corredor Biológico de la Costa Central del Perú - Base de Datos. Tomo I.

Tovar, O. 1993. Las Gramíneas (Poáceas) del Perú. Ruizia. Tomo 13. Madrid. $481 \mathrm{pp}$.

Wittaker, R.H. 1975. Communities and ecosystems. Macmillan Publishing Co., Inc 


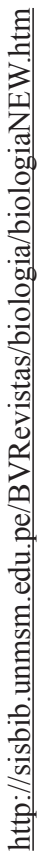

